EUROPEAN ORGANIZATION FOR NUCLEAR RESEARCH

CERN-EP/98-26

17 February 1998

\title{
Measurement of $\tau$ Polarisation at LEP
}

\author{
The L3 Collaboration
}

\begin{abstract}
Using the data collected with the L3 detector at LEP between 1990 and 1995, corresponding to an integrated luminosity of $149 \mathrm{pb}^{-1}$, the $\tau$ longitudinal polarisation has been measured as a function of the production polar angle using the $\tau$ decays $\tau^{-} \rightarrow \mathrm{h}^{-} \nu_{\tau}\left(\mathrm{h}=\pi, \rho, \mathrm{a}_{1}\right)$ and $\tau^{-} \rightarrow \ell^{-} \bar{\nu}_{\ell} \nu_{\tau}(\ell=\mathrm{e}, \mu)$. From this measurement the quantities $\mathcal{A}_{\mathrm{e}}$ and $\mathcal{A}_{\tau}$, which depend on the couplings of the electron and the $\tau$ to the $\mathrm{Z}$, are determined to be $\mathcal{A}_{\mathrm{e}}=0.1678 \pm 0.0127 \pm 0.0030$ and $\mathcal{A}_{\tau}=0.1476 \pm 0.0088 \pm 0.0062$, consistent with the hypothesis of $\mathrm{e}-\tau$ universality. Under this assumption a value of $\mathcal{A}_{\ell}=0.1540 \pm 0.0074 \pm 0.0044$ is obtained, yielding the value of the effective weak mixing angle $\sin ^{2} \bar{\theta}_{\mathrm{W}}=0.2306 \pm 0.0011$.
\end{abstract}

Submitted to Phys. Lett. B 


\section{Introduction}

Parity violation [1] in the process $\mathrm{e}^{+} \mathrm{e}^{-} \rightarrow \mathrm{Z} \rightarrow \mathrm{f} \bar{f}$ gives rise to non-zero polarisation of the $\mathrm{Z}$ and the fermions even in the case of unpolarised electron and positron beams. The $\tau$ leptons decay inside the detector allowing their polarisation to be measured. The $\tau$ longitudinal polarisation, $\mathcal{P}_{\tau}$, is defined as:

$$
\mathcal{P}_{\tau} \equiv \frac{\sigma_{R}-\sigma_{L}}{\sigma_{R}+\sigma_{L}}
$$

where $\sigma_{R}$ and $\sigma_{L}$ are the cross sections for the production of $\tau^{-}$with positive and negative helicity, respectively ${ }^{1)}$. The dependence of $\mathcal{P}_{\tau}$ on the scattering angle in the improved Born approximation at the $\mathrm{Z}$ pole is given by [2]:

$$
\mathcal{P}_{\tau}(\cos \theta)=-\frac{\mathcal{A}_{\tau}\left(1+\cos ^{2} \theta\right)+2 \mathcal{A}_{\mathrm{e}} \cos \theta}{\left(1+\cos ^{2} \theta\right)+2 \mathcal{A}_{\mathrm{e}} \mathcal{A}_{\tau} \cos \theta},
$$

where $\theta$ is the angle between the $\tau^{-}$and the incoming electron beam directions. The quantities $\mathcal{A}_{\mathrm{e}}$ and $\mathcal{A}_{\tau}$ depend on the neutral current couplings of the electron and the $\tau$ to the Z:

$$
\mathcal{A}_{\ell} \equiv \frac{2 \bar{g}_{\mathrm{A}_{\ell}} \bar{g}_{\mathrm{V}_{\ell}}}{\bar{g}_{\mathrm{A}_{\ell}}^{2}+\bar{g}_{\mathrm{V}_{\ell}}^{2}} \quad ; \quad \ell=\mathrm{e}, \tau,
$$

where $\bar{g}_{\mathrm{A}_{\ell}}$ and $\bar{g}_{\mathrm{V}_{\ell}}$ are the effective axial-vector and vector couplings [3] respectively. According to Equation (2), $\mathcal{A}_{\mathrm{e}}$ and $\mathcal{A}_{\tau}$ can be determined simultaneously from a measurement of $\mathcal{P}_{\tau}(\cos \theta)$. This measurement is done by exploiting the kinematics of the $\tau$ decay products $[2,4-6]$ under the assumption of pure ( $\mathrm{V}-\mathrm{A})$ structure in the $\tau$ decay. The latter is supported by experimental results [7]. The measurement of $\mathcal{A}_{\mathrm{e}}$ and $\mathcal{A}_{\tau}$ tests lepton universality in the neutral current and, together with the measurements of forward-backward asymmetries and cross sections, improves significantly the precision of electroweak parameters. It also gives the relative sign of $\bar{g}_{\mathrm{A}_{\ell}}$ and $\bar{g}_{\mathrm{V}_{\ell}}$.

In the Standard Model [8], Equation (3) provides a precise determination of the effective weak mixing angle $[3], \sin ^{2} \bar{\theta}_{\mathrm{W}}$ :

$$
\mathcal{A}_{\ell}=\frac{2\left(1-4 \sin ^{2} \bar{\theta}_{\mathrm{W}}\right)}{1+\left(1-4 \sin ^{2} \bar{\theta}_{\mathrm{W}}\right)^{2}} \quad ; \quad \ell=\mathrm{e}, \tau .
$$

In this paper, semileptonic $\tau$ decays $\tau^{-} \rightarrow \mathrm{h}^{-} \nu_{\tau}\left(\mathrm{h}=\pi, \rho, \mathrm{a}_{1}\right)^{2)}$ and purely leptonic $\tau$ decays $\tau^{-} \rightarrow \ell^{-} \bar{\nu}_{\ell} \nu_{\tau}(\ell=\mathrm{e}, \mu)$ are analysed using the data collected in 1994 and 1995. The results are combined with our previous published ones, based on 1990 to 1993 data [9]. Other measurements of $\mathcal{P}_{\tau}$ have been performed at LEP [10,11] and at lower energy $\mathrm{e}^{+} \mathrm{e}^{-}$colliders [12].

\section{Data Analysis}

The data used in this analysis were collected at centre-of-mass energies around the Z mass, corresponding to a total integrated luminosity of $80 \mathrm{pb}^{-1}$, where $80 \%$ is on the $\mathrm{Z}$ peak and

\footnotetext{
1) Formulae are given for the decay of the $\tau^{-}$. In the analysis the charge conjugate decays are also used.

${ }^{2)}$ No distinction between charged pions and kaons is made in $\tau^{-} \rightarrow \mathrm{h}^{-} \nu_{\tau}$ decay. Both decay modes are combined in the analysis and referred to as $\tau^{-} \rightarrow \pi^{-} \nu_{\tau}$.
} 
$10 \%$ at energies $\sqrt{s}=m_{\mathrm{Z}}-2 \mathrm{GeV}$ and $\sqrt{s}=m_{\mathrm{Z}}+2 \mathrm{GeV}$ each. They are taken with the L3 detector [13] upgraded with a Silicon Microvertex Detector [14], which improves the measurement of track parameters and vertex determination. We exploit this new detector particularly in the analysis of $\tau^{-} \rightarrow \mathrm{a}_{1}^{-} \nu_{\tau} \rightarrow \pi^{+} \pi^{-} \pi^{-} \nu_{\tau}$ decays.

The $\mathrm{e}^{+} \mathrm{e}^{-} \rightarrow \tau^{+} \tau^{-}(\gamma)$ events are selected by requiring low track multiplicity. The identification of the different $\tau$ decay products is performed separately in each hemisphere of the event, which is defined by the plane perpendicular to the event thrust axis. Electrons, muons and $\mathrm{a}_{1}$ mesons are identified in the central part of the detector, which covers $|\cos \theta|<0.72$. Pions and $\rho$ mesons are also identified in the end-cap regions, extending the angular coverage to $|\cos \theta|<0.94$.

For efficiency and background estimates, Monte Carlo events are generated using the programs KORALZ [15] for $\mathrm{e}^{+} \mathrm{e}^{-} \rightarrow \mu^{+} \mu^{-}(\gamma)$ and $\mathrm{e}^{+} \mathrm{e}^{-} \rightarrow \tau^{+} \tau^{-}(\gamma)$, BHAGENE [16] for $\mathrm{e}^{+} \mathrm{e}^{-} \rightarrow$ $\mathrm{e}^{+} \mathrm{e}^{-}(\gamma)$, DIAG36 [17] for $\mathrm{e}^{+} \mathrm{e}^{-} \rightarrow \mathrm{e}^{+} \mathrm{e}^{-} \mathrm{ff}$, where $\mathrm{ff}$ is $\mathrm{e}^{+} \mathrm{e}^{-}, \mu^{+} \mu^{-}, \tau^{+} \tau^{-}$or q$\overline{\mathrm{q}}$, and JETSET [18] for $\mathrm{e}^{+} \mathrm{e}^{-} \rightarrow \mathrm{q} \overline{\mathrm{q}}(\gamma)$. The Monte Carlo events are passed through a full detector simulation based on the GEANT program [19], which takes into account the effects of energy loss, multiple scattering, showering and time dependent detector inefficiencies. These events are reconstructed with the standard L3 reconstruction program. The number of Monte Carlo events in each process is about ten times larger than the data sample.

\section{Particle Identification}

Electrons are identified by an energy deposit in the electromagnetic calorimeter (BGO) which is electromagnetic in shape and consistent in position and energy with a track in the central tracker. Muons are identified by a track in the muon spectrometer originating from the interaction point with a minimum-ionising particle response in the BGO and the hadron calorimeter. The electron and muon identification efficiencies, which are estimated from Monte Carlo, are shown in Figure 1(a) as functions of the normalised particle energy, $E_{\ell} / E_{\text {beam }}$, where $E_{\ell}$ is the lepton energy and $E_{\text {beam }}$ is the beam energy. The average identification efficiencies are $82 \%$ and $70 \%$ for electrons and muons, respectively.

The identification of $\tau^{-} \rightarrow \pi^{-} \nu_{\tau}, \tau^{-} \rightarrow \rho^{-} \nu_{\tau}$ and $\tau^{-} \rightarrow \mathrm{a}_{1}^{-} \nu_{\tau}$ decays is based on the central tracker and calorimeter information. An algorithm [9] is applied to disentangle overlapping neutral electromagnetic clusters in the vicinity of the impact point of the charged hadron in the BGO. Around the impact point, which is predicted by the central tracker, a hadronic shower, whose shape is nearly energy independent, is subtracted from the energy deposit. Electromagnetic neutral cluster identification criteria are applied to the remaining local maxima of energy deposit. The energies and angles are determined for the accepted electromagnetic neutral clusters. Two distinct neutral clusters form a $\pi^{0}$ if their invariant mass is within 40 $\mathrm{MeV}$ of the $\pi^{0}$ mass. A single neutral cluster is considered a $\pi^{0}$ if its energy exceeds $1 \mathrm{GeV}$. Its transverse energy profile must be consistent with either a single electromagnetic shower or a two-photon hypothesis for which the invariant mass is within $50 \mathrm{MeV}$ of the $\pi^{0}$ mass. The $\tau^{-} \rightarrow \pi^{-} \nu_{\tau}$ identification requires that there are no $\pi^{0}$ and no neutral clusters with energy greater than $0.5 \mathrm{GeV}$ in the vicinity of the $\pi^{-}$.

To identify $\tau^{-} \rightarrow \rho^{-} \nu_{\tau}$ decays, one $\pi^{0}$ is required in the hemisphere. The invariant mass of the $\pi^{-} \pi^{0}$ system must be in the range 0.45 to $1.20 \mathrm{GeV}$ and its energy must be larger than $5 \mathrm{GeV}$. The efficiency for $\tau^{-} \rightarrow \pi^{-} \nu_{\tau}$ and $\tau^{-} \rightarrow \rho^{-} \nu_{\tau}$ identification is estimated from Monte Carlo. The average efficiencies are $66 \%(64 \%)$ and $71 \%$ (61\%) in the barrel (end-caps) for $\pi$ and $\rho$, respectively. 
The $\mathrm{a}_{1}$ meson decays via the channels $\mathrm{a}_{1}^{-} \rightarrow \pi^{-} \pi^{0} \pi^{0}$ (1-prong) and $\mathrm{a}_{1}^{-} \rightarrow \pi^{-} \pi^{+} \pi^{-}$(3-prong) with equal probability. The $\mathrm{a}_{1}$ (1-prong) is identified as a charged track accompanied by two $\pi^{0}$. The invariant mass of the three pion system is required to be greater than $0.45 \mathrm{GeV}$. To identify the $\mathrm{a}_{1}$ in the 3 -prong decay mode three charged tracks and no $\pi^{0}$ are required. The $\tau^{-} \rightarrow \mathrm{a}_{1}^{-} \nu_{\tau}$ identification efficiency, estimated from Monte Carlo, is $36 \%$ for the 1-prong and $45 \%$ for the 3 -prong mode.

Figures $1(\mathrm{~b})$ to $1(\mathrm{~d})$ show the dependence of the hadron identification efficiencies on the kinematic variables used to measure $\mathcal{P}_{\tau}$; these are the normalised pion energy, $E_{\pi} / E_{\text {beam }}$, in the $\tau^{-} \rightarrow \pi^{-} \nu_{\tau}$ decay, the angles $\theta_{\rho}^{*}$ and $\psi_{\rho}^{*}[5]$ for $\tau^{-} \rightarrow \rho^{-} \nu_{\tau}$, where:

$$
\cos \theta_{\rho}^{*}=\frac{4 m_{\tau}^{2}}{m_{\tau}^{2}-m_{\rho}^{2}} \frac{E_{\pi^{-}}+E_{\pi^{0}}}{\sqrt{s}}-\frac{m_{\tau}^{2}+m_{\rho}^{2}}{m_{\tau^{2}}^{2}-m_{\rho}^{2}} \quad ; \quad \cos \psi_{\rho}^{*}=\frac{m_{\rho}}{\sqrt{m_{\rho}^{2}-4 m_{\pi}^{2}}} \frac{E_{\pi^{-}}-E_{\pi^{0}}}{\left|\vec{p}_{\pi^{-}}+\vec{p}_{\pi^{0}}\right|}
$$

and the $\omega_{\mathrm{a}_{1}}$ variable [20] for the $\tau^{-} \rightarrow \mathrm{a}_{1}^{-} \nu_{\tau}$ decay, which is defined in terms of the energies and angles of the three pions coming from the $\mathrm{a}_{1}$ and contains all the information about the polarisation of the $\tau$.

\section{Event Selection and Background Rejection}

Events with at least one identified $\tau$ decay are retained for the measurement of $\mathcal{P}_{\tau}$. Additional criteria are applied to reduce background from Bhabha, dimuon and two-photon events and from cosmic ray muons [9].

The shape of residual Bhabha and dimuon background is estimated from data samples selected by relaxing the requirement on the energy of electrons and muons, respectively, in the hemisphere opposite to the identified one. The distribution of the remaining two-photon background is estimated from Monte Carlo. Its normalisation is determined from data using events with large acollinearity. The background from cosmic muons is estimated from a control sample selected in data by loosening the cut on the distance of the muons to the $\mathrm{e}^{+} \mathrm{e}^{-}$interaction vertex. The background from hadronic $\mathrm{Z}$ decays is negligible. The fraction of misidentified $\tau$ decays in each channel is determined using simulated $\mathrm{e}^{+} \mathrm{e}^{-} \rightarrow \tau^{+} \tau^{-}(\gamma)$ events.

The number of selected decays in each exclusive final state, the selection efficiency and the background fractions are given in Table 1 for each $\tau$ decay mode, for the 1994-1995 data. The total number of $\tau$ decays analysed including the 1990-1993 data is 137092 , corresponding to an integrated luminosity of $149 \mathrm{pb}^{-1}$.

\begin{tabular}{lrrrr}
\hline Channel & Decays & \multicolumn{2}{c}{$\varepsilon(\%)$} & \multicolumn{2}{l}{ Bkg. (\%) } \\
& & in $4 \pi$ & \multicolumn{1}{c}{$\tau$} & non- $\tau$ \\
\hline$\tau^{-} \rightarrow \mathrm{e}^{-} \bar{\nu}_{e} \nu_{\tau}$ & 16300 & 45 & 1.6 & 3.6 \\
$\tau^{-} \rightarrow \mu^{-} \bar{\nu}_{\mu} \nu_{\tau}$ & 13920 & 40 & 0.9 & 4.7 \\
$\tau^{-} \rightarrow \pi^{-} \nu_{\tau}$ & 12104 & 42 & 9.8 & 3.7 \\
$\tau^{-} \rightarrow \rho^{-} \nu_{\tau}$ & 22634 & 38 & 11.0 & 1.0 \\
$\tau^{-} \rightarrow \mathrm{a}_{1}^{-} \nu_{\tau}(1$ prong) & 4172 & 22 & 32.7 & 0.0 \\
$\tau^{-} \rightarrow \mathrm{a}_{1}^{-} \nu_{\tau}$ (3 prong) & 4159 & 27 & 16.7 & 0.0 \\
\hline
\end{tabular}

Table 1: Number of selected decays, selection efficiencies $(\varepsilon)$ and fractions from $\tau$ and non- $\tau$ backgrounds for the $\tau$ decay channels used to measure $\mathcal{P}_{\tau}$. 


\section{Measurement Methods}

The $\tau$ longitudinal polarisation, $\mathcal{P}_{\tau}$, is measured in nine $\cos \theta$ intervals ranging from -0.94 to 0.94 . The angle $\theta$ is approximated by the polar angle of the event thrust axis in the $\tau^{-}$ jet direction. The following methods are used to measure $\mathcal{P}_{\tau}$ : the analysis of the exclusive single $\tau$ decays, the analysis of inclusive hadronic $\tau$ decays, the analysis of acollinearity in $\pi \mathrm{X}$ final states, where $\mathrm{X}$ is any 1-prong $\tau$ decay, and an analytical fit to the energy spectra of the exclusive single $\tau$ decays.

The individual measurements of $\mathcal{P}_{\tau}$ for each $\tau$ decay channel in a given $\cos \theta$ bin are corrected accounting for the statistical correlation when both $\tau$ decays in an event are selected for the polarisation measurement. The resulting values are combined with those from the inclusive analysis taking into account the statistical correlation arising from the overlap between the samples. Finally the combination with the results from the acollinearity method is performed. The correlation matrices used in the combination procedure are obtained with a fast Monte Carlo simulation which provides very high statistics event samples [21].

In order to obtain $\mathcal{A}_{\mathrm{e}}$ and $\mathcal{A}_{\tau}$ as given in Equation (2), the measured values are corrected for QED Bremsstrahlung, $\gamma$ exchange and $\gamma$-Z interference contributions, in bins of $\cos \theta$ using the program ZFITTER [22]. These corrections are small at the $\mathrm{Z}$ peak and reach the level of a few per cent for off-peak data.

Decays with wrongly assigned charge migrate between bins of opposite $\operatorname{sign}$ of $\cos \theta$. The fraction of such events on average is $1.4 \%$ in the barrel and $7.5 \%$ in the end-caps. A correction is applied to the measured value of $\mathcal{P}_{\tau}$ in each $\cos \theta$ interval to account for this effect.

\section{Analysis of Exclusive Single $\tau$ Decays}

This method uses spectra of charged and neutral particles in each $\tau$ decay channel generated by the Monte Carlo program KORALZ [15] for positive and negative $\tau^{-}$helicity. The spectra for the two helicity states obtained after detector simulation and reconstruction are fitted to the data distributions, using a binned maximum likelihood method that accounts for finite statistics both in data and Monte Carlo [23].

For the electron and muon spectra, the normalisation of the Bhabha and dimuon background is left free in the fit, whereas the two-photon and cosmic backgrounds are fixed to the estimated amounts. The non- $\tau$ background is fixed in the fit of hadron spectra. The background from other $\tau$ decays is included in the distributions of each helicity and varied in the fit simultaneously with the signal ones.

The spectra of the charged decay products for the channels $\tau^{-} \rightarrow \mathrm{e}^{-} \bar{\nu}_{e} \nu_{\tau}, \tau^{-} \rightarrow \mu^{-} \bar{\nu}_{\mu} \nu_{\tau}$, $\tau^{-} \rightarrow \pi^{-} \nu_{\tau}$ and $\tau^{-} \rightarrow \mathrm{a}_{1}^{-} \nu_{\tau}$, using 1994 and 1995 data, are shown in Figure 2. The angular distributions for the $\tau^{-} \rightarrow \rho^{-} \nu_{\tau}$ decays are shown in Figure 3 for the same data set. Good agreement between data and Monte Carlo distributions is observed. Compatible distributions for the 1990 to 1993 data have been already published [9].

\section{Analysis of Inclusive Hadronic $\tau$ Decays}

The measurement of the $\tau$ polarisation from exclusive hadronic $\tau$ decays, in spite of the high sensitivity, suffers from losses induced by the identification of $\pi^{0}$ in $\tau^{-} \rightarrow \rho^{-} \nu_{\tau}$ and $\tau^{-} \rightarrow \mathrm{a}_{1}^{-} \nu_{\tau}$ decays. Most of these events are recovered by selecting an inclusive sample of 1-prong hadronic $\tau$ decays where no explicit identification of $\pi^{0}$ is attempted [10]. Hadronic $\tau$ decays are identified by one charged track matching an energy deposit in the calorimeters not consistent with an 
electromagnetic shower or a minimum-ionising particle. The invariant mass $m_{\mathrm{h}}$ of the charged hadron and all the neutral deposits in a cone of $30^{\circ}$ around the track is calculated and the resulting spectrum is divided in three bins: $m_{\mathrm{h}}<0.3 \mathrm{GeV}, 0.3<m_{\mathrm{h}}<0.9 \mathrm{GeV}, 0.9<m_{\mathrm{h}}<m_{\tau}$ $\mathrm{GeV}$.

The polarisation-sensitive variables $\Theta$ and $\Psi$ are defined as a generalisation of $\cos \theta_{\rho}^{*}$ and $\cos \psi_{\rho}^{*}$ in Equation (5), by replacing $m_{\rho}$ with $m_{\mathrm{h}}$ and $E_{\pi^{0}}$ with the sum of the energies of all the neutral particles measured in the BGO in a $30^{\circ}$ cone around the charged hadron track. The distribution of these variables is shown in Figures 4 and 5, for data and Monte Carlo, for the three mass bins specified. In the first bin, which is mainly populated by $\tau^{-} \rightarrow \pi^{-} \nu_{\tau}$ decays, the polarisation is extracted by fitting the spectrum of $\Theta$ only. In the second and third mass bins, which are populated mainly by $\tau^{-} \rightarrow \rho^{-} \nu_{\tau}$ and $\tau^{-} \rightarrow \mathrm{a}_{1}^{-} \nu_{\tau}$ decays respectively, the polarisation is obtained from a two-dimensional fit to $\Theta$ and $\Psi$.

The statistical correlation between the results from this analysis and those from the exclusive analysis of single $\tau$ decays is $38 \%$ for $\tau^{-} \rightarrow \pi^{-} \nu_{\tau}, 50 \%$ for $\tau^{-} \rightarrow \rho^{-} \nu_{\tau}$ and $6 \%$ for $\tau^{-} \rightarrow \mathrm{a}_{1}^{-} \nu_{\tau}$.

\section{Analysis of Acollinearity in $\pi \mathrm{X}$ Final States}

Using the acollinearity between the $\tau$ decay products to measure $\mathcal{P}_{\tau}$ [24], additional detector information is exploited giving, in particular, almost independent systematic errors. The selected sample consists of 6763 events, collected in 1993 and $1994{ }^{3)}$, with a $\tau^{-} \rightarrow \pi^{-} \nu_{\tau}$ decay recoiling against a 1-prong $\tau$ decay. For these events, the acollinearity is defined as $\xi \equiv \pi-\alpha_{12}$ where $\alpha_{12}$ is the angle between the charged pion track and the track in the opposite hemisphere.

The tracks are required to be within the region of polar angle $|\cos \theta|<0.72$. As an example, the acollinearity spectrum for the 1994 data is shown in Figure 6.

The correlation in the $\mathcal{P}_{\tau}$ measurement between this method and the exclusive analysis of single $\tau$ decays is $30 \%$.

\section{Analytical Fit to Exclusive $\tau$ Decays Energy Spectra}

We have measured the $\tau$ longitudinal polarisation using a completely independent analysis method, based on the comparison of the $\tau$ decay energy spectra with analytical functions. These functions are calculated for all the 1-prong $\tau$ decays, for $\tau$ leptons with positive or negative helicity [25]. Mass effects and initial and final state radiation are included. Selection and detector effects are taken into account by a convolution of the theoretical expressions with the resolution function and correcting for acceptance. The resolution function is extracted from test beam data and from calibration samples selected in data for this purpose.

This method is used to measure $\mathcal{P}_{\tau}$ using the decay channels $\tau^{-} \rightarrow \mathrm{e}^{-} \bar{\nu}_{e} \nu_{\tau}, \tau^{-} \rightarrow \mu^{-} \bar{\nu}_{\mu} \nu_{\tau}$, $\tau^{-} \rightarrow \pi^{-} \nu_{\tau}$ and $\tau^{-} \rightarrow \rho^{-} \nu_{\tau}$. The results obtained with this method are used as a cross-check, especially of the systematics concerning the resolution and calibration of the detectors.

\section{Systematic Errors}

The main sources of systematic errors are the uncertainties in the energy scales of the different subdetectors, uncertainties in the background estimations and possible biases due to the event selection.

\footnotetext{
${ }^{3)}$ In our previous publication [9] only 1991 and 1992 data were used in the acollinearity method.
} 
The scale uncertainties of the BGO and muon spectrometer are estimated by comparing the detector responses for Bhabha and dimuon data with the beam energy. The central tracker momentum scale uncertainty is determined by comparing its momentum measurement with that of the muon spectrometer. At low energy, a cross calibration of the central tracker with the BGO and the muon spectrometer is performed using electrons and muons from $\tau$ decays and two-photon events. The $\pi^{0}$ peak position in the invariant mass of photon pairs is used as an additional constraint to the BGO calibration. The BGO and the muon spectrometer scale uncertainties are estimated to be $0.5 \%$ at low energy and $0.05 \%$ at high energy. The scale uncertainties are interpolated linearly for intermediate energies. This procedure is checked using radiative Bhabha and dimuon events. The momentum scale of the central tracker is verified to $0.5 \%$ from 1 to $45 \mathrm{GeV}$. The hadron calorimeter scale is estimated at low energy by a comparison with the central tracker momentum measurement and at high energy by the peak position of the $\rho$ resonance in the $\pi^{ \pm} \pi^{0}$ invariant mass distribution. The scale uncertainty is estimated to be $1 \%$ in the barrel and $3 \%$ in the end-caps, independent of the hadron energy. For the evaluation of the systematic errors, the responses of the individual subdetectors are varied according to their scale uncertainties using a fast Monte Carlo simulation.

The normalisation of the non- $\tau$ background is varied within its statistical error. The corresponding change of $\mathcal{P}_{\tau}$ is assigned as the systematic error. In the case of $\tau^{-} \rightarrow \mathrm{e}^{-} \bar{\nu}_{e} \nu_{\tau}$ and $\tau^{-} \rightarrow \mu^{-} \bar{\nu}_{\mu} \nu_{\tau}$, the normalisation of Bhabha and dimuon background is a free parameter in the fit and its uncertainty is already included in the statistical error. The systematic error due to background from other $\tau$ decays is estimated by varying the branching fractions of the contributing decay channels within their errors [26].

Particle identification and background rejection are designed to be nearly independent of the energy of the $\tau$ decay products in order to keep polarisation biases to a minimum. This is checked by comparing Monte Carlo energy and momentum distributions for electrons and muons from Bhabha, dimuon and two-photon control data samples. Good agreement between data and Monte Carlo is found. Finally the important selection cuts are varied. The change in the value of $\mathcal{P}_{\tau}$ is assigned as a systematic error. The systematic error due to the uncertainty in the charge confusion correction is negligible.

The theoretical error accounts for uncertainties in the decay radiation for $\tau^{-} \rightarrow \pi^{-} \nu_{\tau}(\gamma)$ and $\tau^{-} \rightarrow \rho^{-} \nu_{\tau}(\gamma)$ and in the modelling of the $\mathrm{a}_{1}$ in $\tau^{-} \rightarrow \mathrm{a}_{1}^{-} \nu_{\tau}$ decays. Structure dependent effects in $\tau^{-} \rightarrow \pi^{-} \nu_{\tau}(\gamma)$ [27] amount to 0.002 in $\mathcal{P}_{\tau}$ for the $\tau^{-} \rightarrow \pi^{-} \nu_{\tau}$ final state. The effect of decay radiation in $\tau^{-} \rightarrow \rho^{-} \nu_{\tau}(\gamma)$ is estimated to be 0.001 . The $\mathrm{a}_{1}$ resonance and the $\mathrm{a}_{1} \rightarrow \rho \pi$ decay are analysed with different theoretical approaches [28]. The differences in the $\mathrm{a}_{1}$ mass and width are propagated to $\mathcal{P}_{\tau}$ leading to a systematic error of 0.010 on the $\tau^{-} \rightarrow \mathrm{a}_{1}^{-} \nu_{\tau}$ result.

The systematic errors on $\mathcal{A}_{\tau}$ and $\mathcal{A}_{\mathrm{e}}$ are obtained by combining the individual ones in quadrature, allowing for correlations and polar angle dependences. They are summarised in Table 2 .

\begin{tabular}{|c|cccc|c|}
\cline { 2 - 6 } \multicolumn{1}{c|}{} & energy scale & background & selection & theory & total \\
\hline $\mathcal{A}_{\tau}$ & 0.0039 & 0.0012 & 0.0046 & 0.0010 & 0.0062 \\
$\mathcal{A}_{\mathrm{e}}$ & 0.0002 & 0.0019 & 0.0023 & 0.0001 & 0.0030 \\
\hline $\mathcal{A}_{\ell}$ & 0.0027 & 0.0010 & 0.0033 & 0.0007 & 0.0044 \\
\hline
\end{tabular}

Table 2: Systematic error on $\mathcal{A}_{\tau}$ and $\mathcal{A}_{\mathrm{e}}$ from different sources. 


\begin{tabular}{|llcc|}
\hline method & channel & $\mathcal{A}_{\tau}$ & $\mathcal{A}_{\mathrm{e}}$ \\
\hline Exclusive & $\tau^{-} \rightarrow \mathrm{e}^{-} \bar{\nu}_{e} \nu_{\tau}$ & $0.121 \pm 0.031$ & $0.257 \pm 0.046$ \\
& $\tau^{-} \rightarrow \mu^{-} \bar{\nu}_{\mu} \nu_{\tau}$ & $0.144 \pm 0.033$ & $0.206 \pm 0.047$ \\
& $\tau^{-} \rightarrow \pi^{-} \nu_{\tau}$ & $0.142 \pm 0.015$ & $0.146 \pm 0.023$ \\
& $\tau^{-} \rightarrow \rho^{-} \nu_{\tau}$ & $0.155 \pm 0.012$ & $0.147 \pm 0.019$ \\
& $\tau^{-} \rightarrow \mathrm{a}_{1}^{-} \nu_{\tau}$ & $0.191 \pm 0.056$ & $0.214 \pm 0.084$ \\
\hline Inclusive & $\tau^{-} \rightarrow \mathrm{h}^{-} \nu_{\tau}$ & $0.152 \pm 0.015$ & $0.185 \pm 0.033$ \\
\hline Acollinearity & $\tau^{-} \rightarrow \pi^{-} \nu_{\tau}$ & $0.111 \pm 0.041$ & $0.128 \pm 0.058$ \\
\hline \hline Analytical & $\tau^{-} \rightarrow \mathrm{e}^{-} \bar{\nu}_{e} \nu_{\tau}$ & $0.139 \pm 0.032$ & \\
& $\tau^{-} \rightarrow \mu^{-} \bar{\nu}_{\mu} \nu_{\tau}$ & $0.130 \pm 0.035$ & \\
& $\tau^{-} \rightarrow \pi^{-} \nu_{\tau}$ & $0.152 \pm 0.017$ & \\
& $\tau^{-} \rightarrow \rho^{-} \nu_{\tau}$ & $0.161 \pm 0.023$ & \\
\hline
\end{tabular}

Table 3: The results on $\mathcal{A}_{\tau}$ and $\mathcal{A}_{\mathrm{e}}$ from 1990 to 1995 data, for the different methods and channels. The errors are statistical only. Note that these values are subject to correlations, which are taken into account for the determination of the final result.

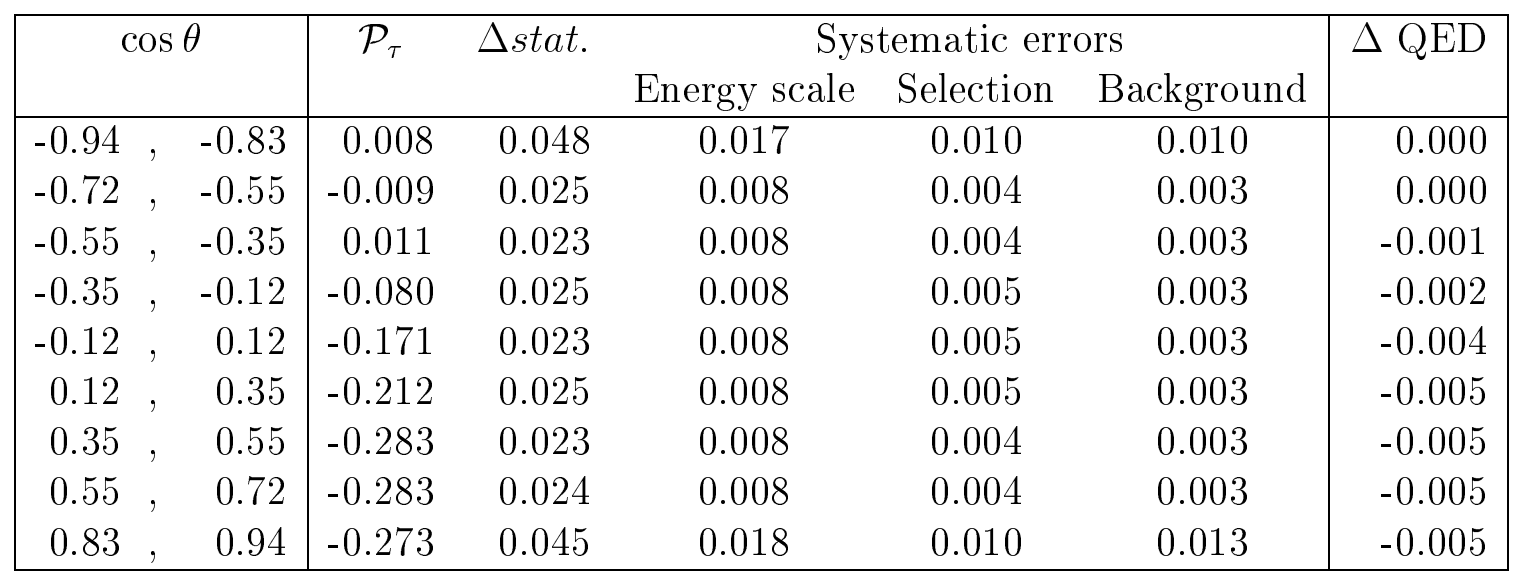

Table 4: Values of $\mathcal{P}_{\tau}$ from 1990 to 1995 data, given as a function of $\cos \theta$, corrected for QED Bremsstrahlung, $\gamma$ exchange and $\gamma-Z$ interference. The correction applied to the measured $\mathcal{P}_{\tau}$ values is shown in the last column.

\section{Results}

The values of $\mathcal{A}_{\tau}$ and $\mathcal{A}_{\mathrm{e}}$ obtained with the different analysis methods and decay channels are shown in Table 3. The results obtained using different analysis methods are in good agreement. Furthermore, the results for the different $\tau$ decay modes agree with each other.

The $\mathcal{P}_{\tau}$ results from 1990 to 1995 data, given in each $\cos \theta$ interval after combining the different methods, are shown in Figure 7 and listed in Table 4 together with the statistical and systematic errors. From a fit to the $\mathcal{P}_{\tau}$ distribution using Equation (2) the final result on $\mathcal{A}_{\tau}$ and $\mathcal{A}_{\mathrm{e}}$ is determined to be:

$$
\begin{aligned}
& \mathcal{A}_{\tau}=0.1476 \pm 0.0088 \text { (stat.) } \pm 0.0062 \text { (sys.) } \\
& \mathcal{A}_{\mathrm{e}}=0.1678 \pm 0.0127 \text { (stat.) } \pm 0.0030 \text { (sys.) }
\end{aligned}
$$

These are consistent with lepton universality. Under this assumption, these results are combined to yield: 


$$
\mathcal{A}_{\ell}=0.1540 \pm 0.0074 \text { (stat.) } \pm 0.0044 \text { (sys.) }
$$

The ratio of the vector to the axial-vector effective couplings is $\bar{g}_{\mathrm{V}_{\ell}} / \bar{g}_{\mathrm{A}_{\ell}}=0.0775 \pm 0.0044$ and the corresponding value of the effective weak mixing angle is:

$$
\sin ^{2} \bar{\theta}_{\mathrm{W}}=0.2306 \pm 0.0011
$$

\section{Acknowledgements}

We wish to express our gratitude to the CERN accelerator division for the excellent performance of the LEP machine. We acknowledge the contributions of all the engineers and technicians who have participated in the construction and maintenance of this experiment.

\section{References}

[1] T. D. Lee and C. N. Yang, Phys. Rev. 104 (1956) 254;

C. S. Wu et al., Phys. Rev. 105 (1957) 1413.

[2] S. Jadach, Z. Wa̧s et al., in 'Z Physics at LEP 1', CERN Report CERN 89-08, eds. G. Altarelli, R. Kleiss and C. Verzegnassi (CERN, Geneva, 1989) Vol. 11, p. 235 and references therein.

[3] A. Sirlin, Phys. Rev. D 22 (1980) 971;

A. A. Akhundov, D. Bardin and T. Riemann, Nucl. Phys. B 276 (1986) 1;

D. Bardin et al., 'Electroweak Working Group Report', CERN Report CERN 95-03, eds. D.Bardin, W. Hollik and G. Passarino, p. 7 and references therein.

[4] Y. S. Tsai, Phys. Rev. D 4 (1971) 2821.

[5] K. Hagiwara, A. D. Martin, D. Zeppenfeld, Phys. Lett. B 235 (1990) 198.

[6] A. Rougé, Z. Phys. C 48 (1990) 75.

[7] ARGUS Collab., H. Albrecht et al., Phys. Lett. B 349 (1995) 576;

ALEPH Collab., D. Buskulic et al., Phys. Lett. B 346 (1995) 379;

L3 Collab., M. Acciarri et al., Phys. Lett. B 377 (1996) 313;

CLEO Collab., J. P. Alexander et al., Phys. Rev. D 56 (1997) 5320.

[8] S.L. Glashow, Nucl. Phys. 22 (1961) 579;

S. Weinberg, Phys. Rev. Lett. 19 (1967) 1264;

A. Salam, "Elementary Particle Theory", Ed. N. Svartholm, Stockholm, "Almquist and Wiksell" (1968), 367.

[9] L3 Collab., M. Acciarri et al., Phys. Lett. B 341 (1994) 245.

[10] DELPHI Collab., P. Abreu et al., Z. Phys. C 67 (1995) 183.

[11] ALEPH Collab., D. Buskulic et al., Z. Phys. C 69 (1996) 183;

OPAL Collab., G. Alexander et al., Z. Phys. C 72 (1996) 365. 
[12] MAC Collab., W. T. Ford et al., Phys. Rev. D 36 (1987) 1971;

CELLO Collab., H. J. Behrend et al., Phys. Lett. B 127 (1983) 270;

AMY Collab., M. H. Lee et al., KEK preprint 90-70 (1990);

VENUS Collab., H. Hanai et al., Phys. Lett. B 403 (1997) 155.

[13] L3 Collab., B. Adeva et al., Nucl. Inst. and Meth. A 289 (1990) 35;

O. Adriani et al., Nucl. Inst. and Meth. A 302 (1991) 53;

K. Deiters et al., Nucl. Inst. and Meth. A 323 (1992) 162.

[14] M. Acciarri et al., Nucl. Inst. and Meth. A 351 (1994) 300.

[15] S. Jadach, B. F. L. Ward and Z. Wạs, Comp. Phys. Comm. 79 (1994) 503.

[16] J. H. Field, Phys. Lett. B 323 (1994) 432;

J. H. Field and T. Riemann, Comp. Phys. Comm 94 (1996) 53.

[17] F. A. Berends, P. H. Daverfeldt and R. Kleiss, Nucl. Phys. B 253 (1985) 441.

[18] T. Sjöstrand, Comp. Phys. Comm. 39 (1986) 347;

T. Sjöstrand and M. Bengtsson, Comp. Phys. Comm. 43 (1987) 367.

[19] The L3 detector simulation is based on GEANT Version 3.15.

See R. Brun et al., "GEANT 3", CERN DD/EE/84-1 (Revised), September 1987.

The GHEISHA program (H. Fesefeldt, RWTH Aachen Report PITHA 85/02 (1985)) is used to simulate hadronic interactions.

[20] M. Davier et al., Phys. Lett. B 306 (1993) 411.

[21] L. Lyons, D. Gibaut and P. Clifford, Nucl. Instr. and Meth. A 270 (1988) 110.

[22] D. Bardin et al., FORTRAN package ZFITTER, and preprint CERN-TH. 6443/92;

D. Bardin et al., Z. Phys. C 44 (1989) 493;

D. Bardin et al., Nucl. Phys. B 351 (1991) 1;

D. Bardin et al., Phys. Lett. B 255 (1991) 290.

[23] R. Barlow and C. Beeston, Comp. Phys. Comm. 77 (1993) 219.

[24] R. Völkert, Ph.D. Thesis, Humboldt University (1997), Interner Bericht, DESY-Zeuthen 97-04, (L3 note 2095);

R. Alemany et al., Nucl. Phys. B 379 (1992) 3.

[25] P. García-Abia, Ph.D. Thesis, Informes Técnicos del CIEMAT 783 (1996), (L3 note 2097).

[26] Particle Data Group, Phys. Rev. D 54 (1996).

[27] R. Decker and M. Finkemeier, Phys. Lett. B 334 (1994) 199.

[28] J. H. Kühn and A. Santamaria, Z. Phys. C 48 (1990) 445;

N. Isgur, C. Morningstar and C. Reader, Phys. Rev. D 39 (1989) 1357. 


\section{The L3 Collaboration:}

M.Acciarri ${ }^{27}$ O.Adriani ${ }^{16}$ M.Aguilar-Benitez ${ }^{26}$ S.Ahlen $^{11}{ }^{1}$ J.Alcaraz ${ }^{26}$ G.Alemanni ${ }^{22}$ J.Allaby, ${ }^{17}$ A.Aloisio ${ }^{29}$ M.G.Alviggi ${ }^{29}$ G.Ambrosi, ${ }^{19}$ H.Anderhub, ${ }^{49}$ V.P.Andreev ${ }^{38}$ T.Angelescu, ${ }^{13}$ F.Anselmo, ${ }^{9}$ A.Arefiev ${ }^{28}$ T.Azemoon, T.Aziz, ${ }^{10}$ P.Bagnaia, ${ }^{37}$ L.Baksay, ${ }^{44}$ R.C.Ball, ${ }^{3}$ S.Banerjee, ${ }^{10}$ Sw.Banerjee, ${ }^{10}$ K.Banicz, ${ }^{46}$ A.Barczyk ${ }^{49,47}$ R.Barillère ${ }^{17}$ L.Barone ${ }^{37}$ P.Bartalini ${ }^{22}$ A.Baschirotto, ${ }^{27}$ M.Basile, ${ }^{9}$ R.Battiston ${ }^{34}$ A.Bay ${ }^{22}$ F.Becattini, ${ }^{16}$ U.Becker, ${ }^{15}$ F.Behner, ${ }^{49}$ J.Berdugo, ${ }^{26}$ P.Berges ${ }^{15}$ B.Bertucci ${ }^{34}$ B.L.Betev, ${ }^{49}$ S.Bhattacharya ${ }^{10}$ M.Biasini ${ }^{34}$ A.Biland ${ }^{49}$ G.M.Bilei ${ }^{34}$ J.J.Blaising, S.C.Blyth, ${ }^{35}$ G.J.Bobbink, R.Bock, A.Böhm, ${ }^{1}$ L.Boldizsar, ${ }^{14}$ B.Borgia, ${ }^{37}$ D.Bourilkov, ${ }^{49}$ M.Bourquin, ${ }^{19}$ D.Boutigny, ${ }^{4}$ S.Braccini, ${ }^{19}$ J.G.Branson, ${ }^{40}$ V.Brigljevic, ${ }^{49}$ I.C.Brock ${ }^{35}$ A.Buffini, ${ }^{16}$ A.Buijs ${ }^{45}$ J.D.Burger ${ }^{15}$ W.J.Burger, ${ }^{34}$ J.Busenitz, ${ }^{44}$ X.D.Cai ${ }^{15}$ M.Campanelli, ${ }^{9}$ M.Capell ${ }^{15}$ G.Cara Romeo, G.Carlino, ${ }^{29}$ A.M.Cartacci ${ }^{16}$ J.Casaus ${ }^{26}$ G.Castellini ${ }^{16}$ F.Cavallari ${ }^{37}$ N.Cavallo, ${ }^{29}$ C.Cecchi ${ }^{19}$ M.Cerrada ${ }^{26}$ F.Cesaroni ${ }^{23}$ M.Chamizo ${ }^{26}$ Y.H.Chang, ${ }^{51}$ U.K.Chaturvedi, ${ }^{18}$ S.V.Chekanov, ${ }^{31}$ M.Chemarin, ${ }^{25}$ A.Chen ${ }^{51}$ G.Chen, ${ }^{7}$ G.M.Chen ${ }^{7}$ H.F.Chen ${ }^{20}$ H.S.Chen ${ }^{7}$ M.Chen, ${ }^{15}$ G.Chiefari, ${ }^{29}$ C.Y.Chien ${ }^{5}$ L.Cifarelli, ${ }^{39}$ F.Cindolo, ${ }^{9}$ C.Civinini, ${ }^{16}$ I.Clare, ${ }^{15}$ R.Clare ${ }^{15}$ H.O.Cohn ${ }^{32}$ G.Coignet, ${ }^{4}$ A.P.Colijn, ${ }^{2}$ N.Colino, ${ }^{26}$ S.Costantini $^{8}$ F.Cotorobai ${ }^{13}$ B.de la Cruz ${ }^{26}$ A.Csilling, ${ }^{14}$ T.S.Dai ${ }^{15}{ }^{2}$ R.D'Alessandro, ${ }^{16}$ R.de Asmundis ${ }^{29}$ A.Degré, ${ }^{4}$ K.Deiters, ${ }^{47}$ P.Denes, ${ }^{36}$ F.DeNotaristefani, ${ }^{37}$ D.DiBitonto, ${ }^{44}$ M.Diemoz, ${ }^{37}$ D.van Dierendonck, ${ }^{2}$ F.Di Lodovico, ${ }^{49}$ C.Dionisi, ${ }^{37}$ M.Dittmar, ${ }^{49}$ A.Dominguez ${ }^{40}$ A.Doria, ${ }^{29}$ M.T.Dova, ${ }^{18, \sharp}$ E.Drago, ${ }^{29}$ D.Duchesneau, ${ }^{4}$ P.Duinker, ${ }^{2}$ I.Duran,${ }^{41}$ S.Dutta ${ }^{10}$ S.Easo, ${ }^{34}$ Yu.Efremenko, ${ }^{32}$ H.El Mamouni, ${ }^{25}$ A.Engler ${ }^{35}$ F.J.Eppling ${ }^{15}$ F.C.Erné, ${ }^{2}$ J.P.Ernenwein ${ }^{25}$ P.Extermann ${ }^{19}$ M.Fabre, ${ }^{47}$ R.Faccini, ${ }^{37}$ S.Falciano ${ }^{37}$ A.Favara ${ }^{16}{ }^{2}$ J.Fay, ${ }^{25}$ O.Fedin ${ }^{38}$ M.Felcini ${ }^{49}$ B.Fenyi ${ }^{44}$ T.Ferguson ${ }^{35}$ F.Ferroni ${ }^{37}$ H.Fesefeldt, ${ }^{1}$ E.Fiandrini, ${ }^{34}$ J.H.Field ${ }^{19}$ F.Filthaut ${ }^{35}$ P.H.Fisher ${ }^{15}$ I.Fisk ${ }^{40}$ G.Forconi ${ }^{15}{ }^{27}$ L.Fredj ${ }^{19}$ K.Freudenreich ${ }^{49}$ C.Furetta ${ }^{27}$ Yu.Galaktionov, ${ }^{28,15}$ S.N.Ganguli, ${ }^{10}$ P.Garcia-Abia, ${ }^{6}$ S.S.Gau, ${ }^{12}$ S.Gentile, ${ }^{37}$ J.Gerald, ${ }^{5}$ N.Gheordanescu, ${ }^{13}$ S.Giagu, S.Goldfarb, ${ }^{22}$ J.Goldstein, ${ }^{11}$ Z.F.Gong, ${ }^{20}$ A.Gougas, ${ }^{5}$ G.Gratta ${ }^{33}$ M.W.Gruenewald, ${ }^{8}$ V.K.Gupta ${ }^{36}$ A.Gurtu ${ }^{10}$ L.J.Gutay, ${ }^{46}$ D.Haas, ${ }^{6}$ B.Hartmann, ${ }^{1}$ A.Hasan ${ }^{30}{ }^{2}$ D.Hatzifotiadou, ${ }^{9}$ T.Hebbeker, ${ }^{8}$ A.Hervé, ${ }^{17}$ J.Hirschfelder, ${ }^{35}$ W.C.van Hoek ${ }^{31}$ H.Hofer ${ }^{49}$ H.Hoorani ${ }^{35}$ S.R.Hou ${ }^{51}$ G.Hu, ${ }^{5}$ V.Innocente ${ }^{17}$ K.Jenkes ${ }^{1}$ B.N.Jin? ${ }^{7}$ L.W.Jones, ${ }^{3}$ P.de Jong, ${ }^{17}$ I.Josa-Mutuberria, ${ }^{26}$ A.Kasser ${ }^{22}$ R.A.Khan, ${ }^{18}$ D.Kamrad ${ }^{48}$ Yu.Kamyshkov, ${ }^{32}$ J.S.Kapustinsky, ${ }^{24}$ Y.Karyotakis, ${ }^{4}$ M.Kaur ${ }^{18, \diamond}$ M.N.Kienzle-Focacci, ${ }^{19}$ D.Kim ${ }^{37}$ D.H.Kim ${ }^{43}$ J.K.Kim ${ }^{43}$ S.C.Kim,${ }^{43}$ W.W.Kinnison ${ }^{24}$ A.Kirkby ${ }^{33}$ D.Kirkby ${ }^{33}$ J.Kirkby ${ }^{17}$ D.Kiss ${ }^{14}$ W.Kittel ${ }^{31}$ A.Klimentov, ${ }^{15,28}$ A.C.König, ${ }^{31}$ A.Kopp ${ }^{48}$ I.Korolko, ${ }^{28}$ V.Koutsenko, ${ }^{15,28}$ R.W.Kraemer, ${ }^{35}$ W.Krenz, ${ }^{1}$ A.Kunin ${ }^{15,28}$ P.Lacentre ${ }^{48, t, \sharp}$ P.Ladron de Guevara, ${ }^{26}$ G.Landi, ${ }^{16}$ C.Lapoint ${ }^{15}$ K.Lassila-Perini ${ }^{49}$ P.Laurikainen ${ }^{21}$ A.Lavorato ${ }^{39}{ }^{2}$ M.Lebeau, ${ }^{17}$ A.Lebedev, ${ }^{15}$ P.Lebrun, ${ }^{25}$ P.Lecomte, ${ }^{49}$ P.Lecoq ${ }^{17}$ P.Le Coultre ${ }^{49}$ H.J.Lee ${ }^{8}$ C.Leggett, ${ }^{3}$ J.M.Le Goff, ${ }^{17}$ R.Leiste ${ }^{48}$ E.Leonardi ${ }^{37}$ P.Levtchenko, ${ }^{38}$ C.Li ${ }^{20}$ C.H.Lin, ${ }^{51}$ W.T.Lin ${ }^{51}$ F.L.Linde ${ }^{2,17}{ }^{17}$ L.Lista ${ }^{29}$ Z.A.Liu, W.Lohmann ${ }^{48}$ E.Longo, ${ }^{37}$ W.Lu, ${ }^{33}$ Y.S.Lu, K.Lübelsmeyer, ${ }^{1}$ C.Luci ${ }^{37}$ D.Luckey ${ }^{15}$ L.Luminari ${ }^{37}$ W.Lustermann ${ }^{47}$ W.G.Ma ${ }^{20}$ M.Maity, ${ }^{10}$ G.Majumder ${ }^{10}$ L.Malgeri ${ }^{37}$ A.Malinin, ${ }^{28}$ C.Maña ${ }^{26}$ D.Mangeol ${ }^{31}{ }^{2}$ S.Mangla ${ }^{10}$ P.Marchesini, ${ }^{49}$ A.Marin ${ }^{11}$ J.P.Martin ${ }^{25}$ F.Marzano ${ }^{37}$ G.G.G.Massaro, D.McNally, ${ }^{17}$ S.Mele, ${ }^{17}$ L.Merola, ${ }^{29}{ }^{2}$ M.Meschini ${ }^{16}$ W.J.Metzger, ${ }^{31}$ M.von der Mey, ${ }^{1}$ Y.Mi, ${ }^{22}$ D.Migani, A.Mihul ${ }^{13}$ A.J.W.van Mil ${ }^{31}$ H.Milcent ${ }^{17}$ G.Mirabelli, ${ }^{37}$ J.Mnich, ${ }^{17}$ P.Molnar, ${ }^{8}$ B.Monteleoni, ${ }^{16}$ R.Moore ${ }^{3}$ T.Moulik, ${ }^{10}$ R.Mount, ${ }^{33}$ F.Muheim, ${ }^{19}$ A.J.M.Muijs, ${ }^{2}$ S.Nahn, ${ }^{15}$ M.Napolitano, ${ }^{29}$ F.Nessi-Tedaldi, ${ }^{49}$ H.Newman ${ }^{33}$ T.Niessen, 1 A.Nippe ${ }^{22}$ A.Nisati, ${ }^{37}$ H.Nowak, ${ }^{48}$ Y.D.Oh ${ }^{43}$ H.Opitz, G.Organtini ${ }^{37}$ R.Ostonen, ${ }^{21}$ S.Palit ${ }^{12}$ C.Palomares ${ }^{26}$ D.Pandoulas, ${ }^{1}$ S.Paoletti, ${ }^{37}$ P.Paolucci ${ }^{29}$ H.K.Park ${ }^{35}$ I.H.Park ${ }^{43}$ G.Pascale ${ }^{37}$ G.Passaleva,${ }^{17}$ S.Patricelli ${ }^{29}$ T.Paul ${ }^{12}$ M.Pauluzzi ${ }^{34}$ C.Paus ${ }^{17}$ F.Pauss ${ }^{49}$ D.Peach ${ }^{17}$ Y.J.Pei ${ }^{1}$ S.Pensotti ${ }^{27}$ D.Perret-Gallix, B.Petersen ${ }^{31}$ S.Petrak ${ }^{8}$ A.Pevsner, ${ }^{5}$ D.Piccolo, ${ }^{29}$ M.Pierii, ${ }^{16}$ P.A.Piroué ${ }^{36}$ E.Pistolesi, ${ }^{27}$ V.Plyaskin ${ }^{28}$ M.Pohl, ${ }^{49}$ V.Pojidaev ${ }^{28,16}$ H.Postema ${ }^{15}$ N.Produit ${ }^{19}$ D.Prokofiev, ${ }^{38}$ J.Quartieri ${ }^{39}$ G.Rahal-Callot ${ }^{49}{ }^{4}$ N.Raja, ${ }^{10}$ P.G.Rancoita ${ }^{27}$ M.Rattaggi, ${ }^{27}$ G.Raven, ${ }^{40}$ P.Razis, ${ }^{30}$ K.Read ${ }^{32}$ D.Ren ${ }^{49}$ M.Rescigno, ${ }^{37}$ S.Reucroft ${ }^{12}$ T.van Rhee ${ }^{45}$ S.Riemann, ${ }^{48}$ K.Riles, O.Rind, A.Robohm, ${ }^{3}$ J.Rodin ${ }^{35}$ B.P.Roe, ${ }^{3}$ L.Romero, ${ }^{26}$ S.Rosier-Lees, ${ }^{4}$ Ph.Rosselet, ${ }^{22}$ W.van Rossum, S.Roth, J.A.Rubio, ${ }^{17}$ D.Ruschmeier, ${ }^{8}$ H.Rykaczewski, ${ }^{49}$ J.Salicio, ${ }^{17}$ E.Sanchez ${ }^{26}$ M.P.Sanders ${ }^{31}$ M.E.Sarakinos ${ }^{21}$ S.Sarkar, ${ }^{10}$ G.Sauvage, C.Schäfer, ${ }^{1}$ V.Schegelsky, ${ }^{38}$ S.Schmidt-Kaerst, ${ }^{1}$ D.Schmitz, M.Schneegans, N.Scholz, ${ }^{49}$ H.Schopper ${ }^{50}$ D.J.Schotanus, ${ }^{31}$ J.Schwenke, G.Schwering, ${ }^{1}$ C.Sciacca ${ }^{29}{ }^{1}$ D.Sciarrino, ${ }^{19}$ L.Servoli ${ }^{34}{ }^{\text {S.Shevchenko }}{ }^{33}$ N.Shivarov, ${ }^{42}$ V.Shoutko, ${ }^{28}$ J.Shukla, ${ }^{24}$ E.Shumilov, ${ }^{28}$ A.Shvorob, ${ }^{33}$ T.Siedenburg, D.Son, ${ }^{43}$ V.Soulimov, ${ }^{29}$ B.Smith, ${ }^{15}$ P.Spillantini, ${ }^{16}$ M.Steuer, ${ }^{15}$ D.P.Stickland, ${ }^{36}$ H.Stone, ${ }^{36}$ B.Stoyanov, ${ }^{42}$ A.Straessner, ${ }^{1}$ K.Sudhakar, ${ }^{10}$ G.Sultanov ${ }^{18}$ L.Z.Sun ${ }^{20}$ G.F.Susinno, ${ }^{19}$ H.Suter ${ }^{49}$ J.D.Swain ${ }^{18}$ X.W.Tang, ${ }^{7}$ L.Tauscher, ${ }^{6}$ L.Taylor ${ }^{12}$ Samuel C.C.Ting, ${ }^{15}$ S.M.Ting, ${ }^{15}$ S.C.Tonwar, ${ }^{10}$ J.Tóth ${ }^{14}$ C.Tully ${ }^{36}$ H.Tuchscherer ${ }^{44}$ K.L.Tung, ${ }^{7}$ Y.Uchida ${ }^{15}$ J.Ulbrichtt, ${ }^{49}$ U.Uwer ${ }^{17}$ E.Valente, ${ }^{37}$ G.Vesztergombi ${ }^{14}$ I.Vetlitsky ${ }^{28}$ G.Viertel $^{49}{ }^{2}$ M.Vivargent, ${ }^{4}$ S.Vlachos ${ }^{6}$ R.Völkert ${ }^{48}$ H.Vogel ${ }^{35}$ H.Vogt ${ }^{48}$ I.Vorobiev, ${ }^{17,28}$ A.A.Vorobyov, ${ }^{38}$ A.Vorvolakos, ${ }^{30}$ M.Wadhwa, ${ }^{6}$ W.Wallraff, ${ }^{1}$ J.C.Wang, ${ }^{15}$ X.L.Wang ${ }^{20}$ Z.M.Wang ${ }^{20}$ A.Weber, ${ }^{1}$ S.X.Wu, ${ }^{15}$ S.Wynhoff, ${ }^{1}$ J.Xu, ${ }^{11}$ Z.Z.Xu, ${ }^{20}$ B.Z.Yang, ${ }^{20}$ C.G.Yang, X.Y.Yao, ${ }^{7}$ J.B.Ye, ${ }^{20}$ S.C.Yeh, ${ }^{52}$ J.M.You, ${ }^{35}$ An.Zalite ${ }^{38}$ Yu.Zalite, ${ }^{38}$ P.Zemp, ${ }^{49}$ Y.Zeng, Z.Zhang, Z.P.Zhang, ${ }^{20}$ B.Zhou, ${ }^{11}$ Y.Zhou, ${ }^{3}$ G.Y.Zhu, R.Y.Zhu, ${ }^{33}$ A.Zichichi, ${ }^{9,17,18}$ F.Ziegler. 
1 I. Physikalisches Institut, RWTH, D-52056 Aachen, FRG ${ }^{\S}$

III. Physikalisches Institut, RWTH, D-52056 Aachen, FRG ${ }^{\S}$

2 National Institute for High Energy Physics, NIKHEF, and University of Amsterdam, NL-1009 DB Amsterdam, The Netherlands

3 University of Michigan, Ann Arbor, MI 48109, USA

4 Laboratoire d'Annecy-le-Vieux de Physique des Particules, LAPP,IN2P3-CNRS, BP 110, F-74941

Annecy-le-Vieux CEDEX, France

5 Johns Hopkins University, Baltimore, MD 21218, USA

6 Institute of Physics, University of Basel, CH-4056 Basel, Switzerland

7 Institute of High Energy Physics, IHEP, 100039 Beijing, China ${ }^{\triangle}$

8 Humboldt University, D-10099 Berlin, FRG $^{\S}$

9 University of Bologna and INFN-Sezione di Bologna, I-40126 Bologna, Italy

10 Tata Institute of Fundamental Research, Bombay 400 005, India

11 Boston University, Boston, MA 02215, USA

12 Northeastern University, Boston, MA 02115, USA

13 Institute of Atomic Physics and University of Bucharest, R-76900 Bucharest, Romania

14 Central Research Institute for Physics of the Hungarian Academy of Sciences, H-1525 Budapest 114, Hungary ${ }^{\ddagger}$

15 Massachusetts Institute of Technology, Cambridge, MA 02139, USA

16 INFN Sezione di Firenze and University of Florence, I-50125 Florence, Italy

17 European Laboratory for Particle Physics, CERN, CH-1211 Geneva 23, Switzerland

18 World Laboratory, FBLJA Project, CH-1211 Geneva 23, Switzerland

19 University of Geneva, CH-1211 Geneva 4, Switzerland

20 Chinese University of Science and Technology, USTC, Hefei, Anhui 230 029, China $\triangle$

21 SEFT, Research Institute for High Energy Physics, P.O. Box 9, SF-00014 Helsinki, Finland

22 University of Lausanne, CH-1015 Lausanne, Switzerland

23 INFN-Sezione di Lecce and Universitá Degli Studi di Lecce, I-73100 Lecce, Italy

24 Los Alamos National Laboratory, Los Alamos, NM 87544, USA

25 Institut de Physique Nucléaire de Lyon, IN2P3-CNRS,Université Claude Bernard, F-69622 Villeurbanne, France

26 Centro de Investigaciones Energeticas, Medioambientales y Tecnologicas, CIEMAT, E-28040 Madrid, Spainb

27 INFN-Sezione di Milano, I-20133 Milan, Italy

28 Institute of Theoretical and Experimental Physics, ITEP, Moscow, Russia

29 INFN-Sezione di Napoli and University of Naples, I-80125 Naples, Italy

30 Department of Natural Sciences, University of Cyprus, Nicosia, Cyprus

31 University of Nijmegen and NIKHEF, NL-6525 ED Nijmegen, The Netherlands

32 Oak Ridge National Laboratory, Oak Ridge, TN 37831, USA

33 California Institute of Technology, Pasadena, CA 91125, USA

34 INFN-Sezione di Perugia and Universitá Degli Studi di Perugia, I-06100 Perugia, Italy

35 Carnegie Mellon University, Pittsburgh, PA 15213, USA

36 Princeton University, Princeton, NJ 08544, USA

37 INFN-Sezione di Roma and University of Rome, "La Sapienza", I-00185 Rome, Italy

38 Nuclear Physics Institute, St. Petersburg, Russia

39 University and INFN, Salerno, I-84100 Salerno, Italy

40 University of California, San Diego, CA 92093, USA

41 Dept. de Fisica de Particulas Elementales, Univ. de Santiago, E-15706 Santiago de Compostela, Spain

42 Bulgarian Academy of Sciences, Central Lab. of Mechatronics and Instrumentation, BU-1113 Sofia, Bulgaria

43 Center for High Energy Physics, Korea Adv. Inst. of Sciences and Technology, 305-701 Taejon, Republic of Korea

44 University of Alabama, Tuscaloosa, AL 35486, USA

45 Utrecht University and NIKHEF, NL-3584 CB Utrecht, The Netherlands

46 Purdue University, West Lafayette, IN 47907, USA

47 Paul Scherrer Institut, PSI, CH-5232 Villigen, Switzerland

48 DESY-Institut für Hochenergiephysik, D-15738 Zeuthen, FRG

49 Eidgenössische Technische Hochschule, ETH Zürich, CH-8093 Zürich, Switzerland

50 University of Hamburg, D-22761 Hamburg, FRG

51 National Central University, Chung-Li, Taiwan, China

52 Department of Physics, National Tsing Hua University, Taiwan, China

$\S$ Supported by the German Bundesministerium für Bildung, Wissenschaft, Forschung und Technologie

$\mp$ Supported by the Hungarian OTKA fund under contract numbers T14459, T19181 and T24011.

b Supported also by the Comisión Interministerial de Ciencia y Technología

\# Also supported by CONICET and Universidad Nacional de La Plata, CC 67, 1900 La Plata, Argentina

七 Supported by Deutscher Akademischer Austauschdienst.

$\diamond$ Also supported by Panjab University, Chandigarh-160014, India

$\triangle$ Supported by the National Natural Science Foundation of China. 

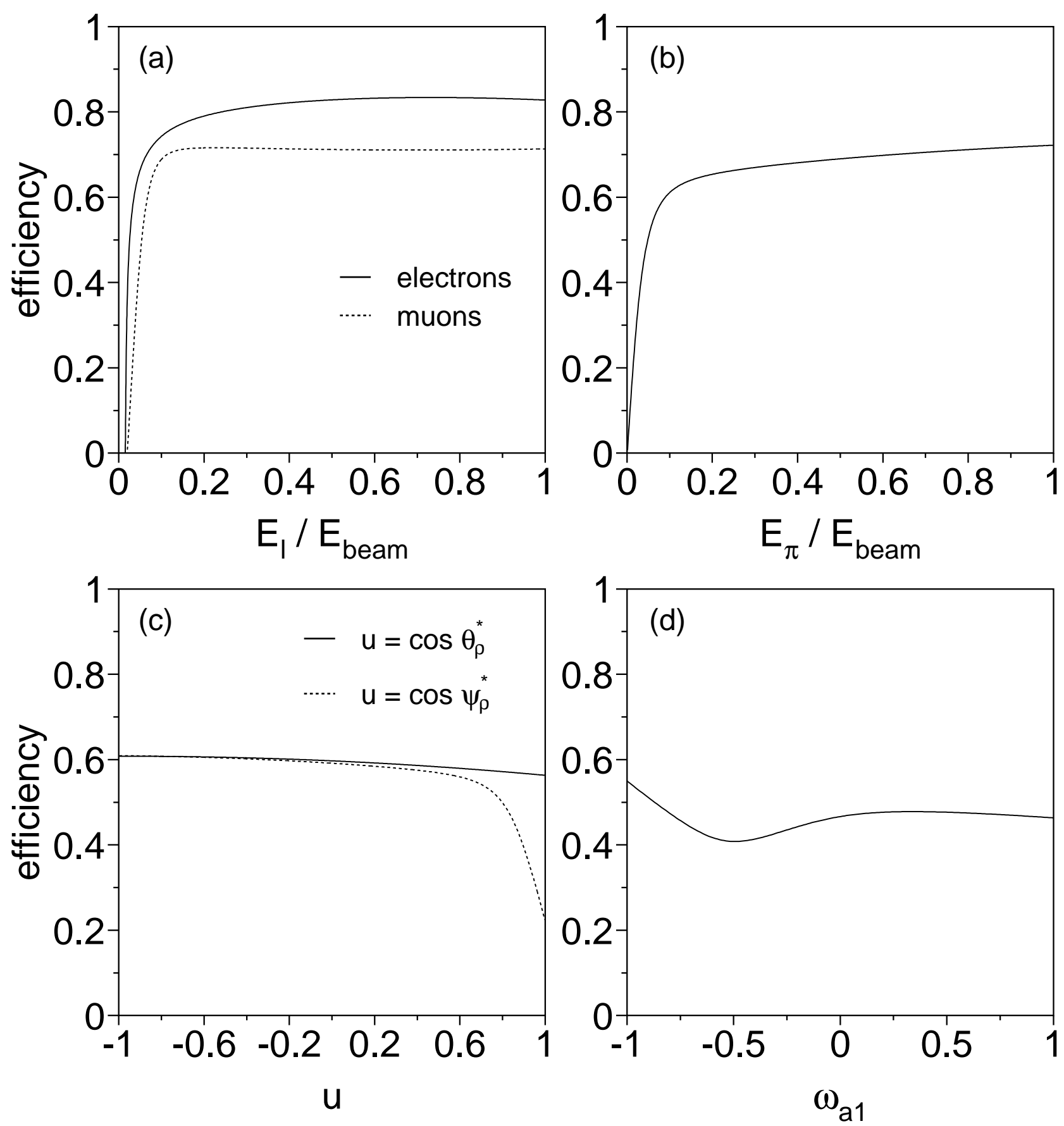

Figure 1: Identification efficiencies for the different $\tau$ decay channels as a function of the corresponding polarisation-sensitive variables described in the text: (a) $\tau^{-} \rightarrow \mathrm{e}^{-} \bar{\nu}_{e} \nu_{\tau}$ and $\tau^{-} \rightarrow \mu^{-} \bar{\nu}_{\mu} \nu_{\tau}$, (b) $\tau^{-} \rightarrow \pi^{-} \nu_{\tau},(\mathrm{c}) \tau^{-} \rightarrow \rho^{-} \nu_{\tau}$ and (d) $\tau^{-} \rightarrow \mathrm{a}_{1}^{-} \nu_{\tau}$ (3-prong). 

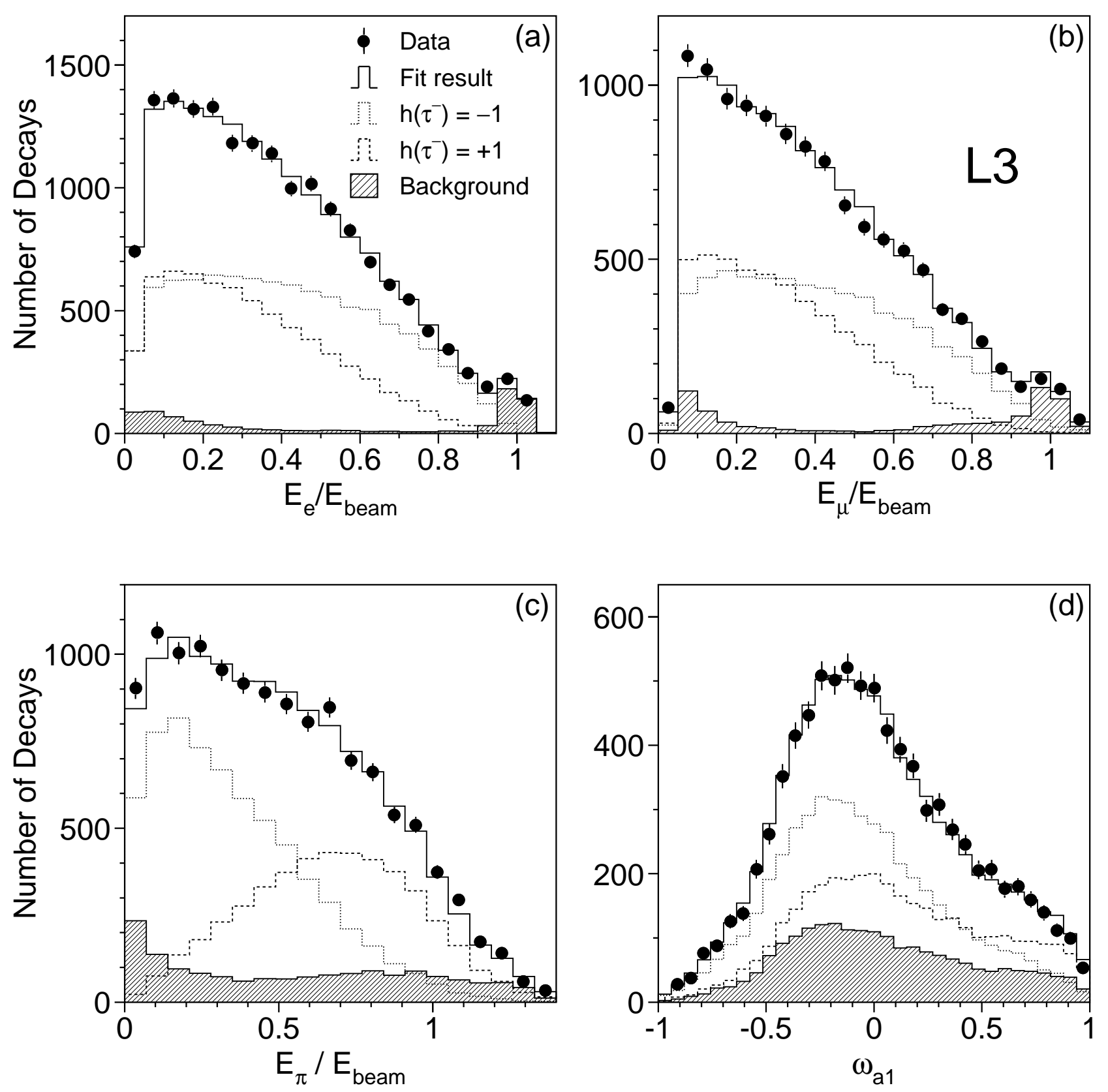

Figure 2: The measured spectra for the polarisation-sensitive variables described in the text for the $\tau$ decaying to (a) electron, (b) muon, (c) $\pi$ and (d) $\mathrm{a}_{1}$ mesons. The data are compared to the results of the fits. The two helicity components and the background are shown separately. 


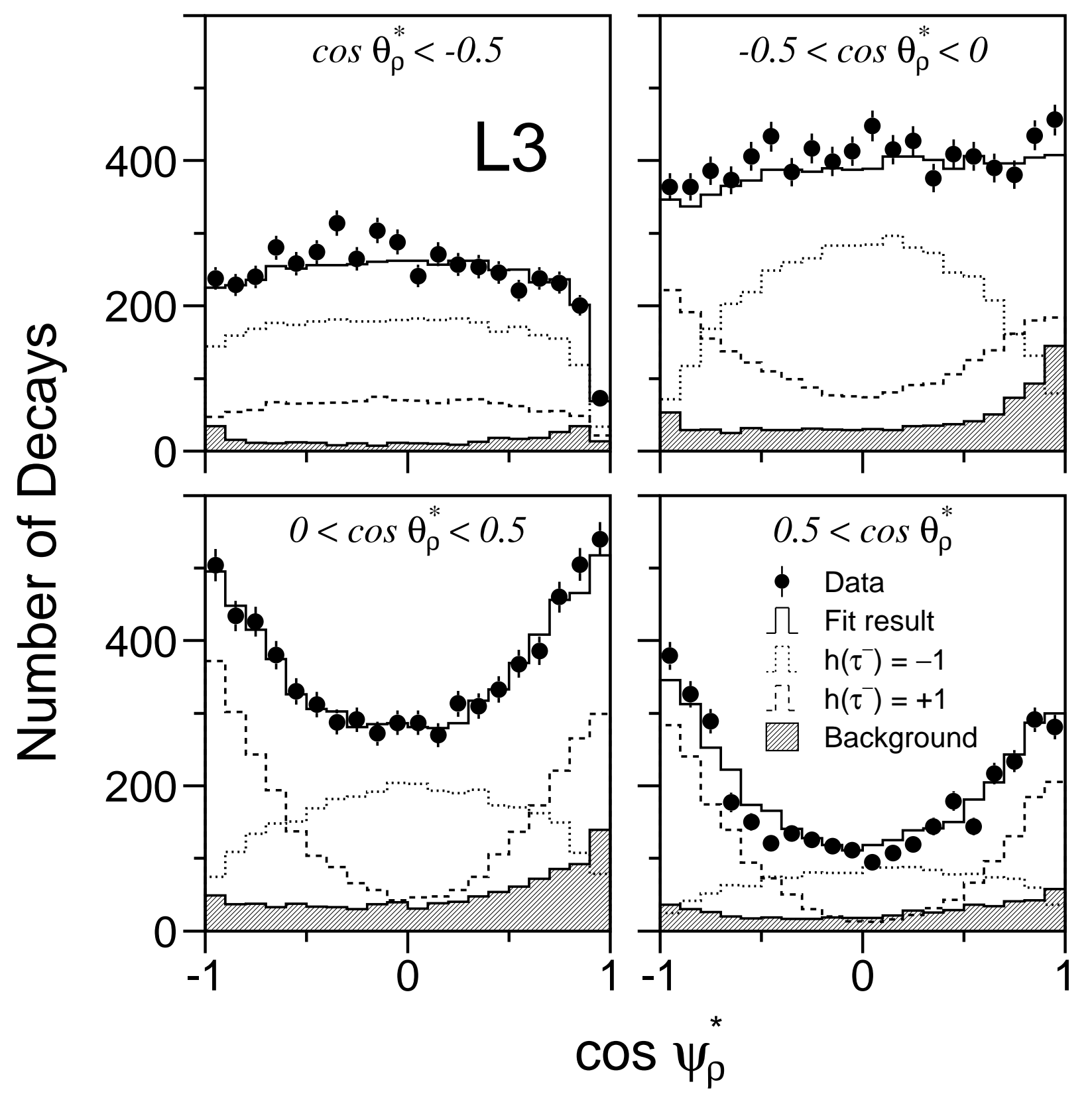

Figure 3: The data spectrum of the $\cos \psi_{\rho}^{*}$ distribution in four slices of $\cos \theta_{\rho}^{*}$ for the $\tau^{-} \rightarrow \rho^{-} \nu_{\tau}$ decays. 


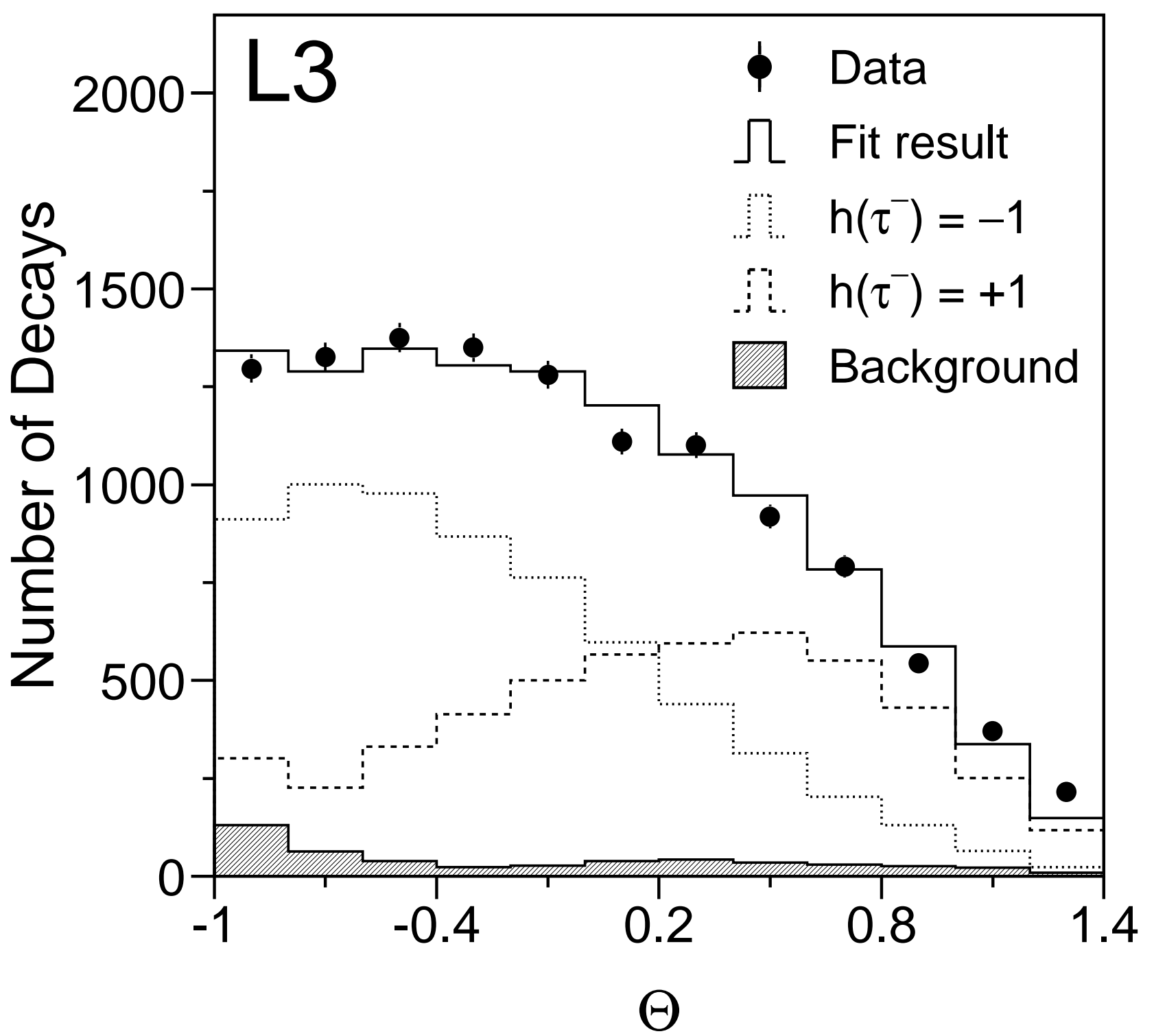

Figure 4: The distribution $\Theta$ of the semileptonic $\tau$ decays used in the inclusive analysis for the first mass bins: $m_{\mathrm{h}}<0.3 \mathrm{GeV}$. 


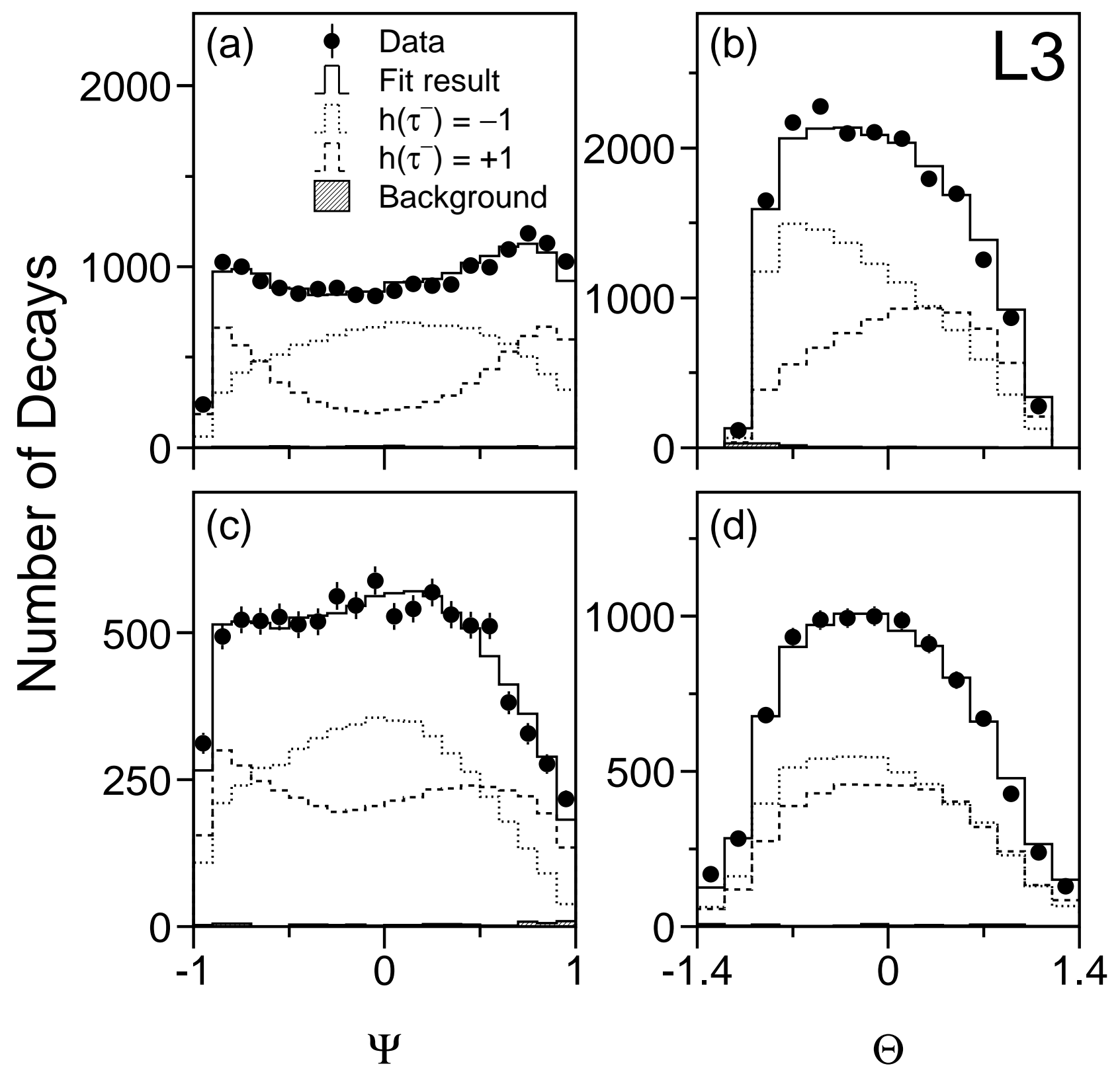

Figure 5: The distributions $\Theta$ and $\Psi$ of the semileptonic $\tau$ decays used in the inclusive analysis for the second and third mass bins: (a,b) $0.3<m_{\mathrm{h}}<0.9 \mathrm{GeV}$ and (c,d) $0.9<m_{\mathrm{h}}<m_{\tau} \mathrm{GeV}$. 


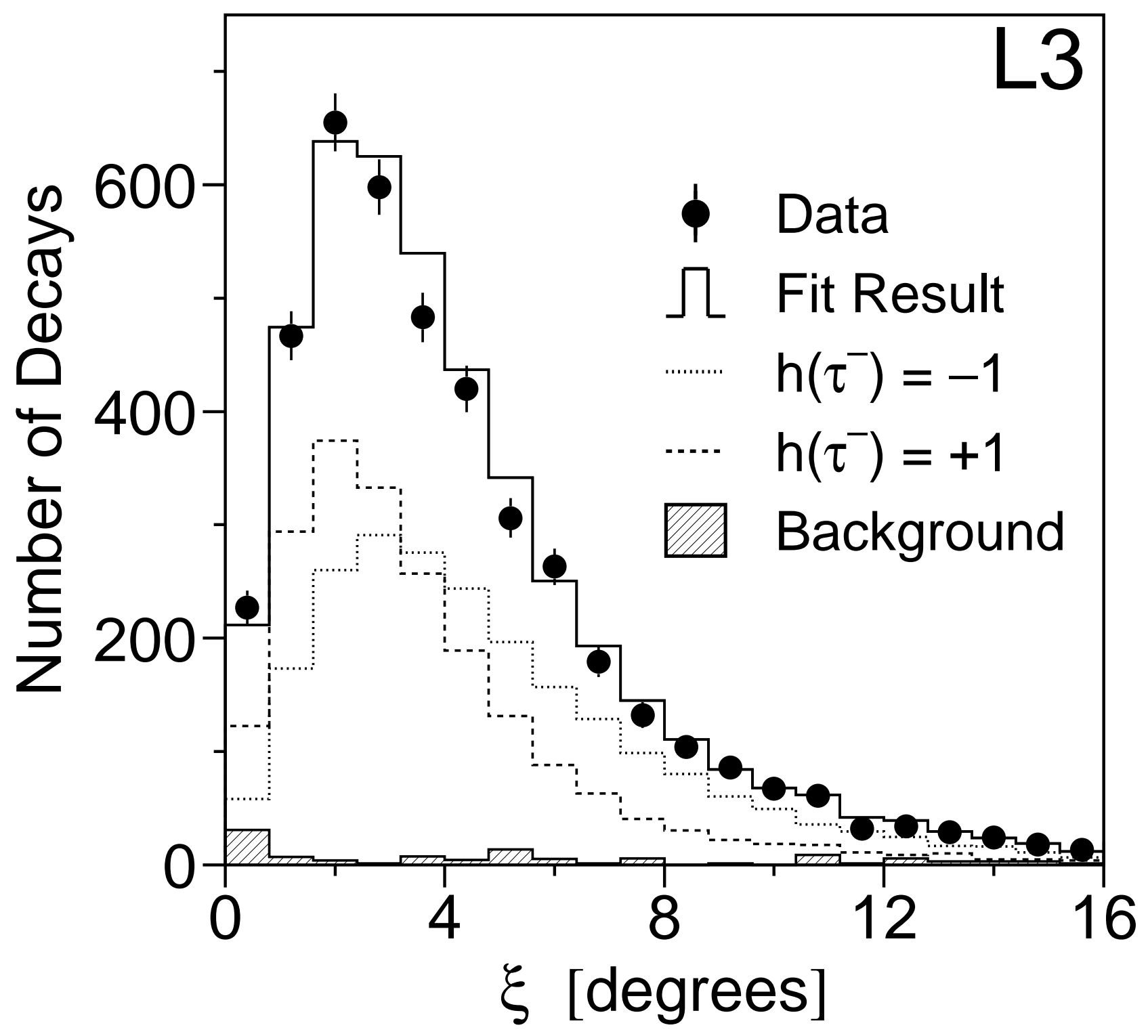

Figure 6: Acollinearity spectrum obtained in 1994 for $\tau^{-} \rightarrow \pi^{-} \nu_{\tau}$ decays recoiling against a 1-prong $\tau$ decay. 


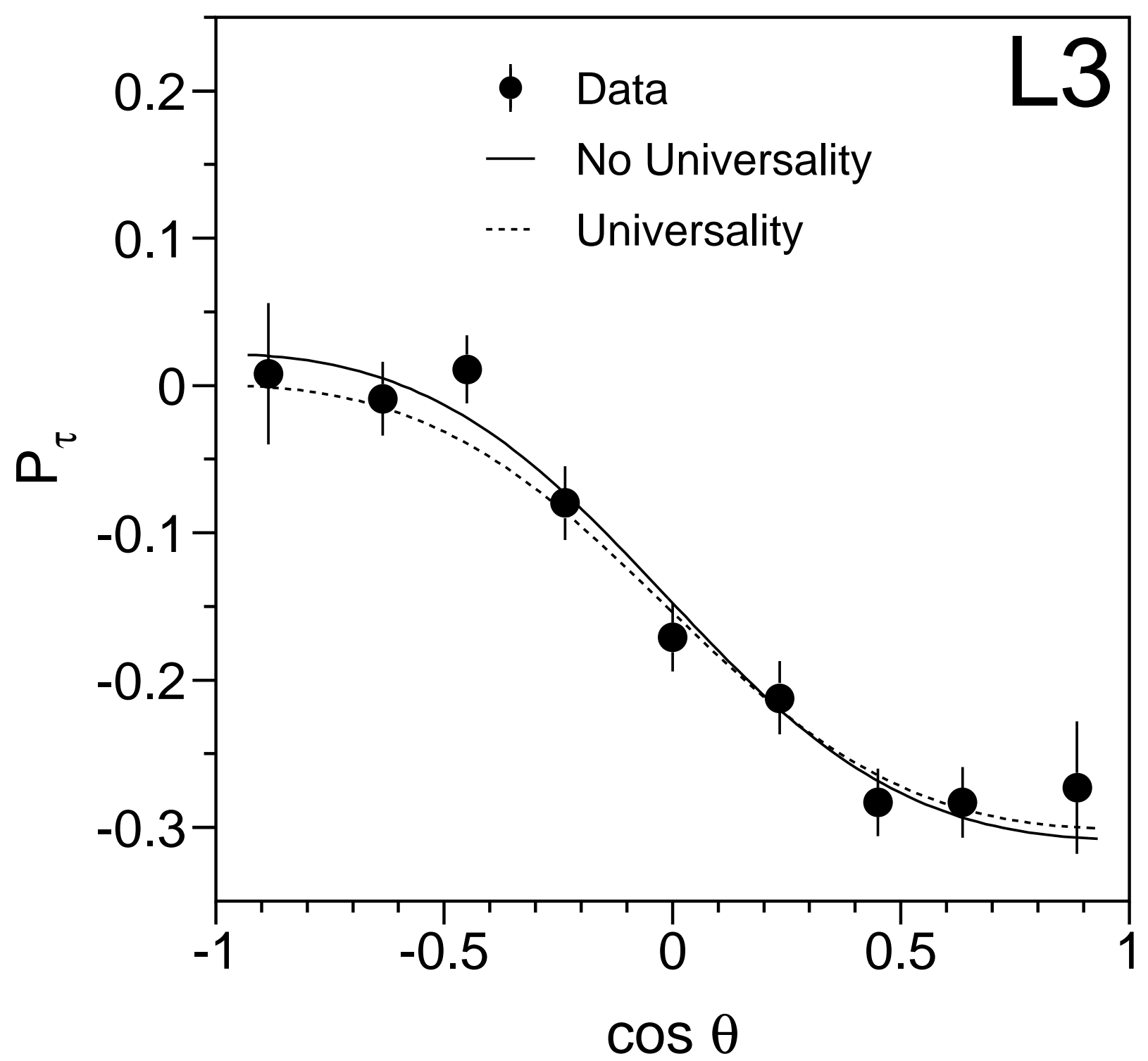

Figure 7: The longitudinal polarisation of the $\tau$ lepton $\mathcal{P}_{\tau}$ as a function of the cosine of the polar angle of the $\tau^{-}$. Included are the data from 1990 to 1995. The solid line corresponds to the two parameter fit of $\mathcal{A}_{\mathrm{e}}$ and $\mathcal{A}_{\tau}$ and the dashed line to the one parameter fit of $\mathcal{A}_{\ell}$ under the assumption of lepton universality. 